\title{
Antiplatelet Therapy for Prevention of Thromboembolic Complications Associated with Coil Embolization of Unruptured Cerebral Aneurysms
}

Yasushi Matsumoto, ${ }^{1}$ Ryushi Kondo, ${ }^{1}$ Yasuhiko Matsumori, ${ }^{1}$ Hiroaki Shimizu, ${ }^{2,3}$ Akira Takahashi ${ }^{2}$ and Teiji Tominaga ${ }^{3}$

1 Department of Neuroendovascular Therapy, Kohnan Hospital, Sendai, Japan

2 Department of Neuroendovascular Therapy, Tohoku University Graduate School of Medicine, Sendai, Japan

3 Department of Neurosurgery, Tohoku University Graduate School of Medicine, Sendai, Japan

\section{Abstract}

\begin{abstract}
Background: Antiplatelet agents are used during endovascular treatment of cerebral aneurysms to prevent thromboembolic complications.

Objective: The aim of this study was to investigate the efficacy of clopidogrel for the prevention of thromboembolic complications during elective coil embolization of unruptured cerebral aneurysms.
\end{abstract}

Methods: Sixty-three patients prospectively received oral clopidogrel $75 \mathrm{mg} /$ day from 3 days before and for 1 day after the procedure at our institute (Kohnan Hospital, Sendai, Japan) during 2007.

Results: At 24 hours post-coiling, significantly less high-intensity areas, detected by MRI with diffusion-weighted imaging (MRI-DWI), were observed in clopidogrel-treated patients compared with a historical control cohort of aspirin (acetylsalicylic acid)-treated patients (13/63 [20.6\%] vs 27/69 [39.1\%]; $\mathrm{p}=0.02$ ), primarily due to a statistically significantly lower rate during repair of small $(<10 \mathrm{~mm})$ lesions $(\mathrm{p}=0.008)$. Also, the rate of periprocedural thromboembolic events was lower in the clopidogrel than the aspirin cohort $(2 / 63$ [3.2\%] vs 5/69 [7.2\%]; $\mathrm{p}=0.3)$.

Conclusions: Clopidogrel was generally well tolerated with no signs of hemorrhagic complications or liver dysfunction.

\section{Background}

Intracranial aneurysms are reported to occur in $1-10 \%$ of the population and are associated with considerable morbidity and mortality fol- lowing rupture. ${ }^{[1-3]}$ The estimated rate of aneurysm rupture ranges between $0-2 \%$ per year, and is dependent on factors such as family history and the size and location of the aneurysm; small aneurysms $(<10 \mathrm{~mm}$ in diameter $)$ in arteries in the 
front of the brain carry a lower risk than those in arteries at the rear of the brain. ${ }^{[3-5]}$

Since its introduction in clinical practice in the 1990s, endovascular coiling for the treatment of cerebral aneurysms has gained widespread use. ${ }^{[4,6]}$ Noninvasive coil embolization for an unruptured aneurysm is relatively safe compared with invasive surgical treatment such as aneurysmal clipping. ${ }^{[3,4]}$ The structure of the platinum coil adjacent to the intimal surface of the artery facilitates the reconstruction of the parent artery by stimulating endothelial growth that promotes stasis, platelet adhesion, clotting, thrombosis, and occlusion of the aneurysm, resulting in blood flow remodeling. ${ }^{[7]}$

Improvements in techniques and management in recent years have facilitated a reduction in procedural risks associated with coil embolization for unruptured cerebral aneurysms; ${ }^{[6,8]}$ however, acute and delayed thromboembolic events, ${ }^{[9]}$ including stroke and transient ischemic attacks (TIA), remain the most common clinical complications ${ }^{[6,10]}$ with reported incidence rates of 4-28\%. ${ }^{[11,12]} \mathrm{Im}$ portantly, the incidence of thromboembolic events associated with aneurysm coiling does not appear to be related to the size and location of the aneurysm, the number of coils used, patient age, or timing of embolization, and it has been suggested that anticoagulation may play a pivotal role in prevention. ${ }^{[13]}$ However, heparin alone has been shown to be limited in preventing thromboembolic events following aneurysm coiling. ${ }^{[13]}$

Aspirin (acetylsalicylic acid) and clopidogrel (Plavix) are used in the management of elective endovascular treatment of cerebral aneurysms to prevent thromboembolic complications despite a lack of robust data to support this approach. ${ }^{[14,15]}$ Although aspirin has shown efficacy in reducing the risk of intraoperative atherothrombotic complications, the antiplatelet agent is associated with insufficient inhibition of platelet aggregation under shear stress, and an increased risk of gastrointestinal bleeding. ${ }^{[16,17]}$ Clopidogrel may be a favorable alternative to aspirin as it has demonstrated greater efficacy in reducing thromboembolic events and less safety issues in patients with vascular disease. ${ }^{[18]}$

The majority of thromboembolic complications associated with endovascular procedures occur perioperatively, which coincides with the period of maximal local prothrombotic activity, i.e. the initial 24 hours; antiplatelet therapy initiated before and/or during intervention may diminish thrombus formation..$^{[9,13,19]}$ Therefore, in this current historical control study, we sought to compare the efficacy of clopidogrel with that of aspirin for reduction in risk of periprocedural thromboembolic complications resulting from elective coil embolization for unruptured cerebral aneurysms by evaluating abnormal high-intensity areas (HIA) diagnostic of ischemic lesions, i.e. restricted diffusion or silent ischemia, at 24 hours after the procedure.

\section{Methods}

Prospective data from the use of clopidogrel during coil embolization for unruptured cerebral aneurysms, collected from January 2007 through to December 2007 (clopidogrel was approved in Japan in 2006 and 2007 for use in stroke and acute coronary syndromes [ACS], respectively), were compared with retrospective data on the use of aspirin for the same procedure collected from February 2005 to December 2006. This study was conducted at Kohnan Hospital, Sendai, Japan, and the local ethics committee provided approval prior to study initiation.

Eligible patients included those with signs and symptoms of suspected cerebral aneurysm who were evaluated and, following confirmation with imaging using either CT or MRI, were scheduled to undergo elective coil embolization for an unruptured cerebral aneurysm. Study inclusion was dependent on full clinical assessments including health status and life expectancy. Informed consent was required prior to the procedure. Data were collected on patient history of previous aneurysms (ruptured or unruptured). Exclusion criteria included allergy or intolerance to aspirin, clopidogrel or platinum, contraindication for MRI, e.g. pacemaker/implantable cardioverter defibrillator or any other metal implants within the body.

All patients underwent diagnostic angiography prior to intervention at which time aneurysm size and location were ascertained. Before the procedure, patients received anticoagulation with intravenous 
heparin 5000 units and during the procedure, heparin 1000 units/hour for a targeted activated clotting time of 200 seconds.

Patients prospectively received clopidogrel $75 \mathrm{mg}$ /day beginning 3 days prior to, and for 1 day following coiling. The historical control cohort comprised consecutive patients who had received oral aspirin $100 \mathrm{mg} /$ day according to the same schedule during the period 2005-6, prior to the approval of clopidogrel. The dosages of aspirin and clopidogrel in this study are those approved for use in stroke or for maintenance therapy of ACS in Japan.

Coil embolization procedures were performed with suitable guiding catheters, microcatheters and coils for each patient under general anesthesia by a neuroanesthesiologist. Balloon neck plasty was also performed, if necessary, for wide neck aneurysms.

Information used from patient charts included date of birth, date of procedure, number of previous aneurysms, aneurysm size, antiplatelet therapy and timing of use (before and/or during intervention) and results of follow-up angiography. Post-procedure, patients were taken to a neurological suite for recovery and neurological status and symptoms were monitored by an independent neurologist.

The primary efficacy endpoints were periprocedural thromboembolic events, which were evaluated as thrombus formation and neurological deficits, either TIA or permanent. Abnormal HIA were detected by MRI examination with diffusionweighted imaging (DWI) [MRI-DWI] at 24 hours after coil embolization using a 3T-MRI scanner (General Electric Company, Fairfield, CT, USA). Images were read in a blinded manner by two specialists in neuroendovascular therapy who were board-certified in Japan. For patient background data, between-group differences were assessed by the $\chi^{2}$ test. Outcomes were also compared with a $\chi^{2}$ test. Statistical calculations were performed using a standard statistical software package (Statemate 2.0; GraphPad Software, Inc., San Diego, CA, USA). Differences in results were considered to be statistically significant if the p-value was $<0.05$.

\section{Results}

Retrospective analysis of data from our institute identified 69 consecutive patients, 16 males and 53 females, who had received aspirin, while during the prospective analysis, 63 consecutive patients, 20 males and 43 females, received clopidogrel treatment for endovascular coil embolization of an unruptured cerebral aneurysm; the evaluable population comprised 132 patients of mean age 59 years. Baseline patient characteristics and aneurysm location and size did not differ significantly between treatment groups (table I). The majority of aneurysms in both cohorts were located in the internal carotid artery and were $<10 \mathrm{~mm}$ in size.

The incidence of HIA assessed by MRI-DWI at 24 hours after coiling was significantly lower with clopidogrel than aspirin $(20.6 \%$ vs $39.1 \%$; $\mathrm{p}=0.02$ ) [figure 1]; ischemic lesions were detected in 13/63 clopidogrel-treated compared with $27 / 69$ aspirin-treated patients. Notably, the rate of HIA occurrence was statistically significantly lower in clopidogrel- than aspirin-treated patients for small $(<10 \mathrm{~mm})$ lesions $(8 / 54$ [14.8\%] vs $22 / 60$ [36.7\%]; $\mathrm{p}=0.008)$, while for larger $(\geq 10 \mathrm{~mm})$ lesions, the rate was also markedly reduced $(3 / 9$ [33.3\%] vs $5 / 9$ [55.6\%]); however, statistical significance was not shown although this may have been due to the small size of these cohorts (figure 2).

Table I. Baseline patient characteristics

\begin{tabular}{|c|c|c|c|}
\hline Parameter & $\begin{array}{l}\text { Aspirin } \\
\text { (acetylsalicylic acid) } \\
100 \mathrm{mg} / \text { day }(\mathrm{n}=69)\end{array}$ & $\begin{array}{l}\text { Clopidogrel } \\
75 \mathrm{mg} / \text { day } \\
(\mathrm{n}=63)\end{array}$ & $\overline{p-V a l u e}$ \\
\hline $\begin{array}{l}\text { Mean age [y] } \\
\text { (range) }\end{array}$ & $58.9(24-85)$ & $59.0(36-79)$ & \\
\hline \multicolumn{4}{|l|}{ Gender [n (\%)] } \\
\hline Female & $53(77)$ & $43(68)$ & NS \\
\hline Male & $16(23)$ & $20(32)$ & \\
\hline \multicolumn{4}{|c|}{ Aneurysm location (n) } \\
\hline ICA & 43 & 40 & NS \\
\hline MCA & 2 & 6 & \\
\hline ACA & 6 & 4 & \\
\hline VA-BA & 17 & 11 & \\
\hline Others & 1 & 2 & \\
\hline \multicolumn{4}{|c|}{ Aneurysm size $(\mathrm{mm})$} \\
\hline$<10$ & 60 & 54 & NS \\
\hline$\geq 10$ & 9 & 9 & \\
\hline
\end{tabular}




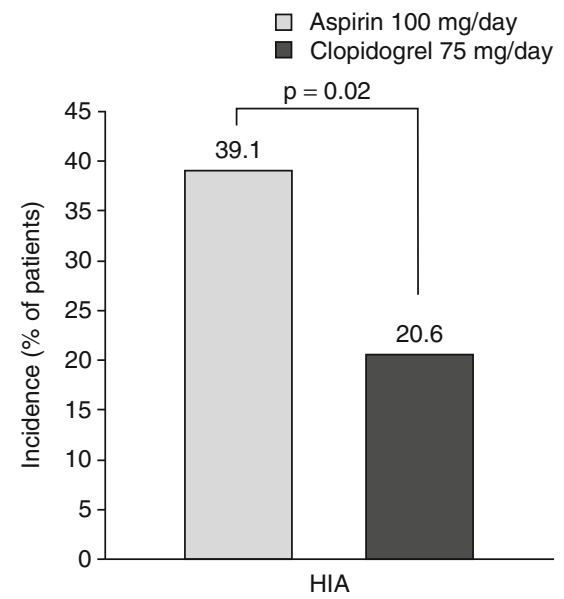

Fig. 1. Incidence of high-intensity areas (HIA) assessed by MRI with diffusion-weighted imaging at 24 hours post-coil embolization for unruptured cerebral aneurysm following aspirin (acetylsalicylic acid) or clopidogrel treatment.

Assessment of the occurrence of symptomatic TIA or stroke showed that compared with aspirin treatment, the rate of periprocedural thromboembolic events was lower in the cohort that received clopidogrel (2/63 [3.2\%] vs 5/69 [7.2\%]; p=0.30) [figure 3]. Unfortunately, one patient in the clopidogrel-treated group had hemiparesis following the procedure, but other patients showed no signs of symptomatic infarction, even in the presence of a lesion found by MRI-DWI.

An example case is shown in figure 4. An unruptured anterior communicating artery aneurysm was treated by coil embolization with clopidogrel treatment. Clot formation occurred in the parent arteries during coiling. Percutaneous transluminal angioplasty was performed immediately and the clot was subsequently cleared away. Even though MRI-DWI revealed a small lesion at the right frontal lobe on day 1 post-procedure, the patient had no neurologic deficits.

Clopidogrel was well tolerated in patients undergoing coil embolization for unruptured cerebral aneurysm; no hemorrhagic complications or bleeding were identified and there were no signs of liver dysfunction, as assessed by biochemical laboratory blood assays.

\section{Discussion}

This study showed a greater antithrombotic effect with clopidogrel than with aspirin treatment (historical control) in patients undergoing elective coil embolization for an unruptured cerebral aneurysm. The incidence of abnormal HIA assessed by MRI-DWI at 24 hours after coiling was significantly lower with clopidogrel than with aspirin treatment $(\mathrm{p}=0.02)$, and there were less periprocedural thromboembolic events with clopidogrel, although this was not statistically significant $(\mathrm{p}=0.30)$.

Management guidelines recommend surgical or endovascular intervention for the treatment of an unruptured intracranial aneurysm. ${ }^{[20-22]}$ It is well established that thromboembolic events are the most common complications arising during or after aneurysm coiling. ${ }^{[1,12]}$ Both the catheter and the coil mass are a potential source for thrombus formation where clotting may occur. ${ }^{[11,13,14]}$

A number of studies that used MRI-DWI for detection of early ischemia associated with Guglielmi detachable coils for the treatment of unruptured

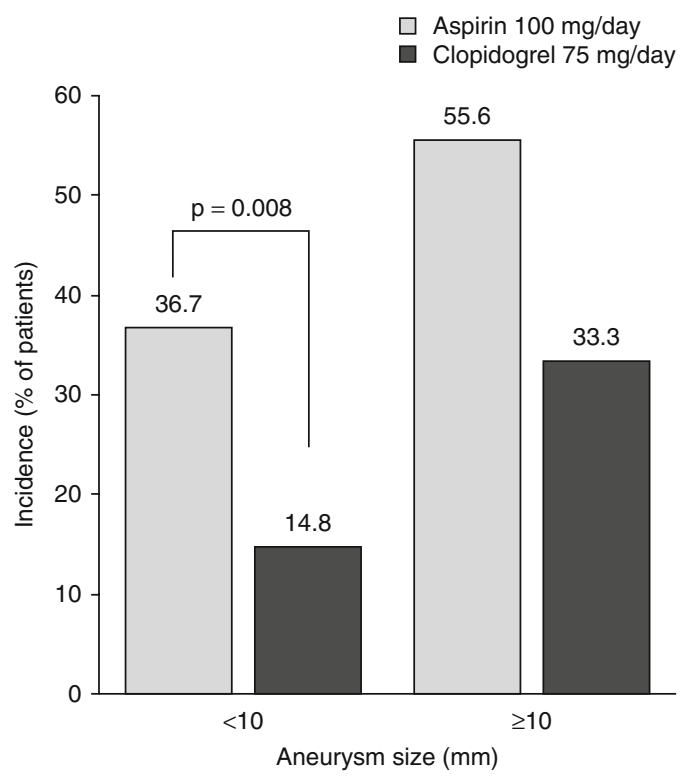

Fig. 2. Frequency of high-intensity areas by aneurysm size (< or $\geq 10 \mathrm{~mm}$ ) at 24 hours post-coil embolization for unruptured cerebral aneurysm following aspirin (acetylsalicylic acid) or clopidogrel treatment. 


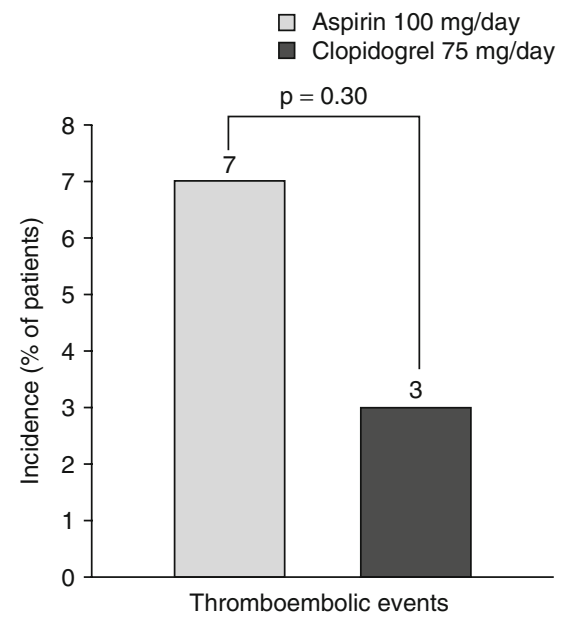

Fig. 3. Incidence of periprocedural thromboembolic events.

cerebral aneurysms showed a high rate of silent thromboembolic events, $42 \%,{ }^{[13]} 49 \%,{ }^{[23]}$ and $61 \% .{ }^{[11]}$ Although subsequent clinical outcomes of such events are rare, ${ }^{[11]}$ antiplatelet agents are a safe option for reduction in the risk of thromboembolic events, asymptomatic or symptomatic. ${ }^{[11,13]}$

In one study, compared with no antiplatelet therapy, there was a significant reduction in thromboembolic events with intravenous aspirin (8.8\% vs $17.6 \% ; \mathrm{p}=0.028$ ) with no increase in intraoperative bleeding during endovascular treatment of 247 patients with ruptured or unruptured cerebral aneurysms. ${ }^{[14]}$ Similarly, a retrospective analysis of 10-year data from 369 patients who underwent elective coil embolization of unruptured cerebral aneurysms showed that compared with no antiplatelet treatment (16\%), the rate of symptomatic thromboembolic events was significantly lower in patients who received oral aspirin and/or clopidogrel treatment $(2 \% ; \mathrm{p}=0.004)$, especially when administered pre- and post-procedure versus only post-procedure (1.9\% vs $2.3 \%) \cdot{ }^{[15]}$ In addition, recent retrospective analyses of trials of oral antiplatelet treatment (clopidogrel, ${ }^{[24,25]}$ aspirin, ${ }^{[25]}$ or both ${ }^{[25]}$ ) given prior to, but not following, coil embolization of unruptured cerebral aneurysms showed trends in reduction $(7.4 \% \text { vs } 12.6 \% ; \mathrm{p}=0.05)^{[24]}$ or significant $(2.1 \% \text { vs } 12.8 \% ; \mathrm{p}=0.023)^{[25]}$ reductions in perioperative thromboembolic complications, with no increase in hemorrhagic complication rates. It must be noted that these trials differed in design compared with each other and in comparison with our current trial, both were large trials that provided baseline stratified risks for thromboembolic complications. The availability of new consensus recommendations for reporting standards for endovascular treatment of intracranial aneurysms may facilitate the publication of trial results amenable for direct comparison. ${ }^{[26]}$

Clopidogrel is approved to reduce thrombotic events in a number of indications including ACS, stroke, myocardial infarction, and ischemic heart disorders. The current study highlights the benefits of clopidogrel compared with aspirin for prophylaxis of thromboembolic complications during aneurysm coiling. Although there was no significant between-treatment difference in the incidence of intraoperative clot formation in this study, it is a

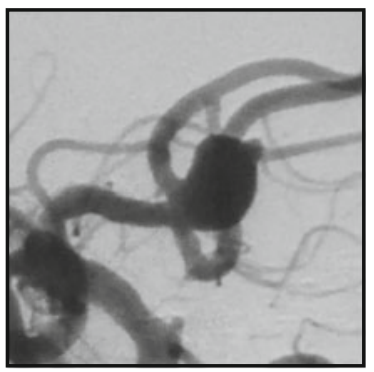

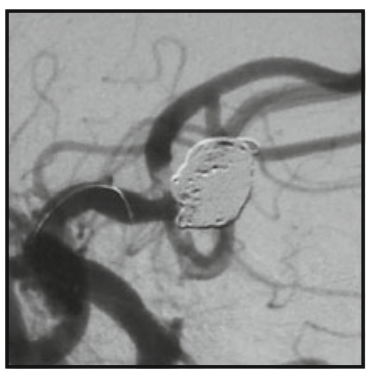

c

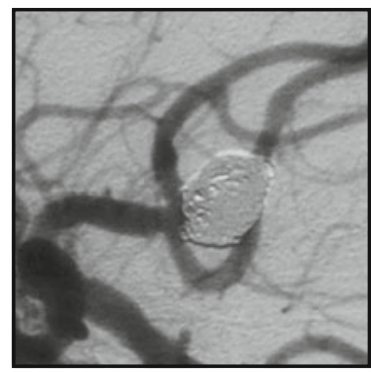

d

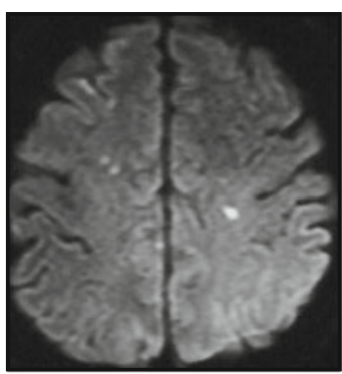

Fig. 4. An unruptured anterior communicating artery aneurysm in digital subtraction angiography (a) before and (b) during coil embolization showing clot formation occurring in both the right and left anterior cerebral artery at the end of the procedure, and (c) following percutaneous transluminal angiography that was performed immediately (the clot was subsequently cleared away). (d) Diffusion-weighted MRI revealed a small lesion at the right frontal lobe on day 1 after the procedure; however, the patient had no neurologic deficits. 
important to reduce such events. In contrast with aspirin, which enhances shear stress-induced platelet aggregation, clopidogrel is known to inhibit shear stress-induced, as well as adenosine diphosphatedependent, platelet aggregation. ${ }^{[19]}$ In this respect, clopidogrel has greater potential to inhibit platelet function more effectively, which may account for the present results. Future studies with larger sample populations may allow potential betweengroup differences to be detected.

Our study is not without limitations, including: a study population derived from both retrospective and prospective data; short-term follow-up; the absence of platelet function assays to assess resistance to antiplatelet treatment; practice effects such as increased operator experience over time or use of balloon- or stent-assisted coil treatment, which may have influenced observed results; and the presence of confounding factors (e.g. patient co-morbidities such as cardiovascular [including smoking history] or atherosclerotic [presence of atherosclerosis/previous stroke] risk factors) that could not be ruled out as influences contributing to thromboembolic events in affected patients.

\section{Conclusion}

The results of our study suggest that clopidogrel is an effective and well tolerated antiplatelet agent in patients undergoing coil embolization of an unruptured cerebral aneurysm. Previous experience with aspirin suggests that clopidogrel may offer superior short-term benefit, which needs to be evaluated in a robustly designed, larger prospective trial that would allow the inclusion of a sufficient number of patients with unruptured cerebral aneurysms, including those with large aneurysms, to derive a statistically definitive result.

\section{Acknowledgments}

The authors would like to thank Nila Bhana, MSc, of inScience Communications, a Wolters Kluwer business, for providing medical writing support funded by sanofi-aventis, Japan.

The authors have no conflicts of interest that are directly relevant to the content of this study.

\section{References}

1. Wanke I, Doerfler A, Dietrich U, et al. Endovascular treatment of unruptured intracranial aneurysms. AJNR Am J Neuroradiol 2002 May; 23 (5): 756-61

2. Meyer FB, Morita A, Puumala MR, et al. Medical and surgical management of intracranial aneurysms. Mayo Clin Proc 1995 Feb; 70 (2): 153-72

3. Wiebers DO, Whisnant JP, Huston 3rd J, et al. Unruptured intracranial aneurysms: natural history, clinical outcome, and risks of surgical and endovascular treatment. Lancet 2003 Jul 12; 362 (9378): 103-10

4. Brisman JL, Song JK, Newell DW. Cerebral aneurysms. N Engl J Med 2006 Aug 31; 355 (9): 928-39

5. Brown Jr RD, Huston J, Hornung R, et al. Screening for brain aneurysm in the Familial Intracranial Aneurysm study: frequency and predictors of lesion detection. J Neurosurg 2008 Jun; 108 (6): 1132-8

6. Lanterna LA, Tredici G, Dimitrov BD, et al. Treatment of unruptured cerebral aneurysms by embolization with guglielmi detachable coils: case-fatality, morbidity, and effectiveness in preventing bleeding - a systematic review of the literature. Neurosurgery 2004 Oct; 55 (4): 767-75; discussion 75-8

7. Ansari SA, Lassig JP, Nicol E, et al. Thrombosis of a fusiform intracranial aneurysm induced by overlapping neuroform stents: case report. Neurosurgery 2007 May; 60 (5): E950-1; discussion E-1

8. van Rooij WJ, Sluzewski M. Procedural morbidity and mortality of elective coil treatment of unruptured intracranial aneurysms. AJNR Am J Neuroradiol 2006 Sep; 27 (8): $1678-80$

9. Qureshi AI, Luft AR, Sharma M, et al. Prevention and treatment of thromboembolic and ischemic complications associated with endovascular procedures: part II - clinical aspects and recommendations. Neurosurgery 2000 Jun; 46 (6): 1360-75; discussion 75-6

10. Bendok BR, Hanel RA, Hopkins LN. Coil embolization of intracranial aneurysms. Neurosurgery 2003 May; 52 (5): 1125-30; discussion 30

11. Rordorf G, Bellon RJ, Budzik Jr RE, et al. Silent thromboembolic events associated with the treatment of unruptured cerebral aneurysms by use of Guglielmi detachable coils: prospective study applying diffusion-weighted imaging. AJNR Am J Neuroradiol 2001 Jan; 22 (1): 5-10

12. Brooks NP, Turk AS, Niemann DB, et al. Frequency of thromboembolic events associated with endovascular aneurysm treatment: retrospective case series. J Neurosurg 2008 Jun; 108 (6): 1095-100

13. Grunwald IQ, Papanagiotou P, Politi M, et al. Endovascular treatment of unruptured intracranial aneurysms: occurrence of thromboembolic events. Neurosurgery 2006 Apr; 58 (4): 612-8; discussion 8

14. Ries T, Buhk JH, Kucinski T, et al. Intravenous administration of acetylsalicylic acid during endovascular treatment of cerebral aneurysms reduces the rate of thromboembolic events. Stroke 2006 Jul; 37 (7): 1816-21

15. Yamada NK, Cross 3rd DT, Pilgram TK, et al. Effect of antiplatelet therapy on thromboembolic complications of elective coil embolization of cerebral aneurysms. AJNR Am J Neuroradiol 2007 Oct; 28 (9): 1778-82 
16. Antithrombotic Trialists' Collaboration. Collaborative metaanalysis of randomised trials of antiplatelet therapy for prevention of death, myocardial infarction, and stroke in high risk patients. BMJ 2002 Jan 12; 324 (7329): 71-86

17. Mehta SR, Yusuf S, Peters RJ, et al. Effects of pretreatment with clopidogrel and aspirin followed by long-term therapy in patients undergoing percutaneous coronary intervention: the PCI-CURE study. Lancet 2001 Aug 18; 358 (9281): 527-33

18. A randomised, blinded, trial of clopidogrel versus aspirin in patients at risk of ischaemic events (CAPRIE). CAPRIE Steering Committee. Lancet 1996 Nov 16; 348 (9038): 1329-39

19. Qureshi AI, Luft AR, Sharma M, et al. Prevention and treatment of thromboembolic and ischemic complications associated with endovascular procedures: Part I-Pathophysiological and pharmacological features. Neurosurgery 2000 Jun; 46 (6): 1344-59

20. Bederson JB, Awad IA, Wiebers DO, et al. Recommendations for the management of patients with unruptured intracranial aneurysms : a statement for healthcare professionals from the Stroke Council of the American Heart Association. Circulation 2000 Oct 31; 102 (18): 2300-8

21. Johnston SC, Higashida RT, Barrow DL, et al. Recommendations for the endovascular treatment of intracranial aneurysms: a statement for healthcare professionals from the Committee on Cerebrovascular Imaging of the American Heart Association Council on Cardiovascular Radiology. Stroke 2002 Oct; 33 (10): 2536-44

22. Meyers PM, Schumacher HC, Higashida RT, et al. Indications for the performance of intracranial endovascular neurointerventional procedures: a scientific statement from the American Heart Association Council on Cardiovascular Radiology and Intervention, Stroke Council, Council on Cardiovascular Surgery and Anesthesia, Interdisciplinary Council on Peripheral Vascular Disease, and Interdisciplinary Council on Quality of Care and Outcomes Research. Circulation 2009 Apr 28; 119 (16): 2235-49

23. Soeda A, Sakai N, Sakai H, et al. Thromboembolic events associated with Guglielmi detachable coil embolization of asymptomatic cerebral aneurysms: evaluation of 66 consecutive cases with use of diffusion-weighted MR imaging. AJNR Am J Neuroradiol 2003 Jan; 24 (1): 127-32

24. Kang HS, Han MH, Kwon BJ, et al. Is clopidogrel premedication useful to reduce thromboembolic events during coil embolization for unruptured intracranial aneurysms? Neurosurgery 2010 Nov; 67 (5): 1371-6; discussion 6

25. Hwang G, Jung C, Park SQ, et al. Thromboembolic complications of elective coil embolization of unruptured aneurysms: the effect of oral antiplatelet preparation on periprocedural thromboembolic complication. Neurosurgery 2010 Sep; 67 (3): 743-8; discussion 8

26. Meyers PM, Schumacher HC, Higashida RT, et al. Reporting standards for endovascular repair of saccular intracranial cerebral aneurysms. AJNR Am J Neuroradiol 2010 January 1; 31 (1): E12-24

Correspondence: Dr Yasushi Matsumoto, Department of Neuroendovascular Therapy, Kohnan Hospital, 4-20-1 Nagamachi-minami, Taihaku-ku, Sendai, 982-8523, Japan. E-mail: matsumoto@kohnan-sendai.or.jp 